\title{
Die Symphonie des Hevein
}

\section{Hevein Symphony}

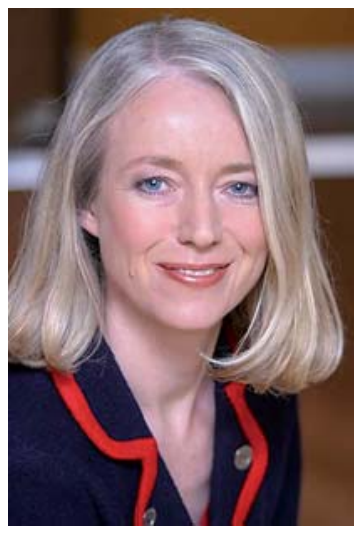

Prof. Dr. Christiane Bayerl
Bibliografie

Dol http://dx.doi.org/

10.1055/s-0030-1256970

Akt Dermatol 2011; 37; 383

(c) Georg Thieme Verlag KG .

Stuttgart · New York

ISSN 0340-2541

Korrespondenzadresse

Prof. Dr. med. Christiane Bayerl Klinik für Dermatologie

und Allergologie

HSK, Wilhelm-Fresenius-Klinik GmbH Städtisches Klinikum Wiesbaden

Lehrkrankenhaus der Univ. Mainz Aukammallee 39

65191 Wiesbaden

christiane.bayer!@

hsk-wiesbaden.de
Immer mehr Kreuzallergene des Latex werden beschrieben. Meist sind es Früchte und Gemüse. Kreuzallergien finden sich bei 20-60\% der LatexSensibilisierten. Die erste Geige spielen Banane, Avokado, Kastanie, Kiwi und Papaya. In der zweiten Reihe sitzen die seltenen Kreuzallergene wie Kartoffel, Tomate, Mango, Melone, Feige, Pfirsich und Buchweizen. Als die beiden führenden Allergene wurden daher das Latex-Hevein (Hev b 6.02) und als Variation über das Latex-Thema die FruchtKlasse-I-Chitinasen mit einer Hevein-ähnlichen Domäne angenommen. Nun zeigte sich aber in einer neuen Studie, dass unter Hevein-sensibilisierten Latexallergikern der Prozentsatz der Nahrungsmittelallergien $(15 / 34=44 \%)$ gleich war $\mathrm{zu}$ dem Latex-allergischer Patienten ohne HeveinSensibilisierung $(11 / 25=44 \%)$ [1]. Also ist wohl Hevein nicht das führende Allergen und nicht das Leitmotiv der Kreuzreaktion.

Darüber hinaus finden sich immer wieder Soloeinlagen seltener Kreuzallergene wie Aubergine und der „Custard apple“ [2], der sogenannte Zimtoder Rahmapfel, der jedoch in unbearbeiteter Form schon den Namen trägt. Er stammt aus der Familie der Anonnaceae und findet sich in der afrikanischen Savanne und in Lateinamerika. Es gibt die Stachel-, die Schuppen- und die Netz-Annonen. Die $200 \mathrm{~g}$ schweren Früchte wachsen an kleinen Bäumen und muten wie Beeren an. Sie haben ein weißes oder orangefarbenes Fleisch und schmecken nach Annanas, Erdbeere oder Birne mit Zimt mit einem etwas rahmigen Geschmack. Affen vertilgen die Frucht sehr gerne. Für den Menschen wird das Fruchtfleisch in der Getränkeindustrie als Aromastoff eingesetzt. Auf den Gourmetseiten finden sich Gaumen-wässernde Rezepturen von Custard-Apple-Eis oder Rahmapfel-Panacotta.

Die „Kastanien aus dem Ofen holen“ - für jemanden anderen - ist ein umgangssprachlicher Ausdruck, der zur Vorsicht wegen der Hitze, aber nicht wegen des Allergengehaltes rät. Häufiger genossen werden Kastanien in der kalten Jahreszeit und sie sind auch sehr gut zum Händewärmen geeignet, wenn man sie noch in der Tüte vom Kastanienröster in den Stadtzentren erhält. Die Hauptallergene der Kastanie (Castanea sativa) sind Cas s 5 (Klasse-I-Chitinase), Cas s 8 (Lipidtransferprotein-Panallergenfamilie) und ein 24kD-Kastanien-Protein, das Homologien zu den Hülsenfrüchten zeigt. Bei den Kreuzallergien zwischen Latex und Kastanien fanden sich die Kreuzreaktionen am häufigsten bei Patienten, die spezifisches IgE gegen Hev b 8 (Profilin) aufwiesen [3]. Profilin ist das Allergen, das bei vielen Pollen, in Früchten und bei Latex gefunden wurde.
Und nun darf ich Ihnen einen neuen Solisten in dem Orchester der Latex-Frucht-Kreuzallergene vorstellen, die „Cassava“ [4]. 2003 erfolgte die erste Publikation zu 3 Patienten mit Kreuzreaktion zwischen „Cassava“ und Latex. „Cassava“ ist auch bekannt als Maniok, Yucca oder Tapioca (Manihot esculenta) und gehört zu den Euphorbiaceae, beheimatet in Westindien, Lateinamerika und Afrika. Maniok wird gekocht, gebacken und als Mehl für Brot, Gebäck und Kuchen eingesetzt. Vor der weiteren Prozessierung muss Maniok geschält werden, um die Hydrocyansäure aus der Rinde loszuwerden, die verantwortlich gemacht wird für neuropathische Schädigungen. Ein brasilianischer und ein afrikanischer Patient mit Maniok- und Latex-Allergie wurden bereits beschrieben. Nun kam zuletzt eine spanische Kasuistik hinzu [4]. Bei der Patientin lag zeitlich zuerst eine Pollinose, dann eine Latexallergie, dann eine Kreuzreaktion auf Früchte und Gemüse (Latex-Frucht-Syndrom) vor. Dann erst folgte die Maniok-Sensibilisierung, die auf beruflicher Exposition beruhte. Die 27-jährige Frau war in einem Restaurant beschäftigt, das kubanische Spezialitäten anbot. Bereits 2 Jahre zuvor hatte sie beim Schälen rohen Manioks Niesen und Rhinorrhoe bemerkt. In den Folgemonaten entwickelt sich eine Urtikaria der Hände und über die Zeit ein Exanthem, Angioödem des Gesichts, Dyspnoe und Dysphonie. Im zweiten Expositionsjahr kamen am Arbeitsplatz Magenschmerzen, Übelkeit und Erbrechen dazu. Avokado und Bananen lösten die gleiche Symptomatik aus und Kondome konnten wegen vaginalem Pruritus nicht mehr verwendet werden.

Soweit zur Globalisierung der Nahrungsmittelallergene!

Christiane Bayerl

\section{Literatur}

1 Radauer C, Adhami F, Fürtler I, Wagner S, Allwandt D, Scala E, Ebner C, Hafner C, Hemmer W, Mari A, Breiteneder $H$. Latex-allergic patients sensitized to the major hevein and hevein-like domains of class I chitinases show no increased frequency of latex-associated plant food allergy. Mol Immunol 2011; 48: 600-609

2 Gamboa PM, Sánchez-Monge R, Díaz-Perales A, Salcedo G, Ansótegui I, Sanz ML. Latex-vegetable syndrome due to custard apple and aubergine: new variations of the hevein symphony. J Invest Allergol Clin Immunol 2005; 15: 308-311

3 Raulf-Heimsoth M, Kespohl S, Crespo JF, Rodriguez J, Feliu A, Brüning T, Rihs HP. Natural rubber latex and chestnut allergy: cross-reactivity or co-sensitization? Allergy 2007; 62: 1277-1281

4 Ibero M, Castillo MJ, Pineda F. Allergy to Cassava: a new allergenic food with cross-reactivity to latex. J Invest Allergol Clin Immunol 2007; 17: 409-412 